\title{
Anti-von Willebrand Factor Nanobody
}

National Cancer Institute

\section{Source}

National Cancer Institute. Anti-von Willebrand Factor Nanobody. NCI Thesaurus. Code

C113796.

A humanized, bivalent anti-von Willebrand factor (VWF) nanobody with potential antiplatelet activity. Anti-von Willebrand factor nanobody specifically binds to the A1 domain of the VWF molecule, thereby inhibiting and neutralizing VWF activity. This prevents the interaction of VWF with the platelet glycoprotein (Gp)Ib-IX-V receptor and prevents VWFmediated platelet aggregation. VWF is a glycoprotein and plays a key role in blood coagulation. Increased VWF, which is seen in a number of diseases, is associated with an increased risk in thrombosis. The nanobody formulation allows for rapid distribution, onset of action and clearance. The nanobody is based on the smallest functional fragments of single-chain antibodies that naturally occur in the Camelidae family. 